DOI: 10.17234/SRAZ.65.26

UDK: 821.133.1.09 Louis, É.

\title{
Les violences du modèle : Édouard Louis
}

\author{
Maja Vukušić Zorica \\ Faculté de Philosophie et Lettres \\ Université de Zagreb \\ mzorica@ffzg.hr
}

La problématisation de la violence, omniprésente dans les romans d'Édouard Louis (En finir avec Eddy Bellegueule, Histoire de la violence et Qui a tué mon père), va devenir ici la plateforme pour la remise en question de la thèse sous-entendue : la vie n'est pas une biographie (Quignard) et l'hybridisation du genre qui joint la fictionnalisation de la biographie et la socioanalyse n'y peut rien, à l'encontre des modèles identifiés : le Retour à Reims de Didier Eribon et les Méditations pascaliennes de Bourdieu. Il y a une violence que Louis dénonce, celle de la société (la « fatalité géographique ») envers les «marginaux », dont les homosexuels, et il y a une violence qu'il perpétue - la violence de toute biographie, la violence de la parole et la violence identitaire. Surgit la question des limites entre l'engagement, l'activisme, le témoignage, l'actuel indéniable et la littérature. La fuite décidément n'est pas le seul projet de Louis.

Mots-clés : Édouard Louis, Didier Eribon, Pierre Bourdieu, violence, honte, hybridisation

\section{Faire école ? - Violence}

Édouard Louis, jeune auteur contemporain né en Picardie en 1992, célèbre pour ses romans En finir avec Eddy Bellegueule (2014), l'Histoire de la violence (2016) et Qui a tué mon père? (2018), dont les deux premiers ont été traduits en croate en 2019, s'impose comme un écrivain engagé depuis son premier «Bildungsroman », En finir avec Eddy Bellegueule. Ce dernier raconte la jeunesse d'un jeune homosexuel en province, isolé et maltraité, qui doit fuir sa vie pour pouvoir s'inventer à Paris. L'Histoire de la violence raconte l'épisode dramatique d'un rendez-vous qui finit par un viol et une tentative de meurtre par strangulation, suivis par une enquête, racontés par la sœur à son mari tandis que le narrateur les épient. Le dernier, Qui a tué mon père ? condamne encore une fois la violence qui détruit les plus vulnérables.

Aussi la violence que Derrida nomme «brutalité » 2018 : 118-119) est-elle le thème central de l'œuvre de Louis. Cette violence, physique ou symbolique, crue 
ou raffinée, est un irréductible - ce que affirment son « maître » Didier Eribon, qui écrit son Retour à Reims (2009, traduit en croate en 2019) en appliquant les préceptes des Méditations pascaliennes de Pierre Bourdieu, dont le but était de dépasser le clivage entre la théorie et la pratique par la critique des positions scolastiques qui transforme la perception du réel social en incorporant le lieu de l'analyse dans la réflexion théorique. Ainsi Bourdieu met-il en évidence le mouvement perpétuel entre la théorie de la pratique et la pratique de la théorie. Tout en essayant d'expliquer la transposition du modèle de la subversion esthétique à la politique (la possibilité pour le mouvement gay et lesbien de se placer à l'avant-garde des réflexions théoriques), Bourdieu synthétisera ses concepts, depuis l'habitus jusqu'au champ et la domination, de l'illusio jusqu'à la violence symbolique, comme deux manières de conceptualiser le réel social, de la connaissance par le corps jusqu'à la connaissance de l'autre par la sociologie. Le rôle crucial de l'espace y surgit comme le lieu de l'inscription des pratiques sociales, qu'après Eribon, va mettre en place Louis.

Louis dénonce ouvertement la société, la « fatalité géographique » (les « effets de lieux » et les « voix absentes » de Bourdieu), le conditionnement et le déterminisme social, la cruauté de la société, dont fait partie aussi la famille, notamment celle des milieux pauvres.

La grille est connue - dès qu'il y a système, il y a des intrus, des exclus ${ }^{1}$. Or, alors que 1'Esquisse d'une auto-analyse, le dernier ouvrage de Bourdieu (2002/2004), avertit contre les illusions de l'individualisme méthodologique et du déterminisme aveugle en rejetant catégoriquement la confession et l'autobiographie, Louis semble prêt à utiliser les concepts bourdieusiens sans les remettre en question ou même exprimer des réticences. La boîte à outils foucaldienne marche toujours.

\section{Le performatif}

Déjà le premier roman, l'histoire d'un jeune homosexuel, victime d'une communauté pauvre et cruelle, doit réussir sa fuite à Paris pour survivre ${ }^{2}$. La deuxième partie du roman, intitulée «L'échec et la fuite », dit expressément que la fuite est venue après une série d'échecs. La fuite n'était pas un projet prémédité, une victoire, une sorte d' " enivrez-vous » à la Daniel Guérin, mais la résignation de ne pas pouvoir être comme les autres autour de lui. Ses essais touchants et triste d'être « un dur » (2014: 182), bref, un homme, montrent que son péché majeur, aux yeux de son milieu, c'est la féminisation.

1 Or, si Eribon et Louis sont des «transfuges de classe » (et heureux de l'être) par rapport aux milieux parisiens intellectuels et bourgeois, ils le sont aussi par rapport à leurs familles, ce dont se rend compte Eribon (2016 : 71-173, 86).

2 Le motif de la fuite est présent dans l'Histoire de la violence aussi (sa fuite devant Reda, la fuite du père de Reda) car elle donne la possibilité, non pas pour un nouveau début, mais pour la réinvention du passé. Selon Louis, le passé est la seule chose qui puisse changer ; le père de Reza avait plus peur du passé que de l'avenir (2016 : 63). 
Plus que le politiquement correct, il y a une autre facette du polemos, qui n'est pas un goût de la guerre ou de la polémique, mais celui défini par la volonté d'établir un champ de bataille, toujours crucialement historique, où « il n'y a pas de métalangage, il n'y a pas de lieu de vérité extérieur au champ » (Derrida, Ferraris 2018 : 18), mais qui néanmoins conditionne l'existence d'une « destinée stratégique, destinée au stratagème par la mise en question de la vérité du champ » (Ibid.).

Aussi s'agit-il de produire «performativement » (Derrida 2018 : 20) un certain contexte, de se créer une position. Louis réussit à créer cette œuvre autant « contextualisée » que « contextualisante » (Ibid. : 21), imbibée des apports théoriques d'Eribon, qui sont aussi performatifs : ils proposent une perception du réel qui finit par construire ce dernier.

Or, Louis répète l'aventure de son maître et de son « livre en guerre » (Eribon 2016 : 60), Retour à Reims. En fait, la guerre qu'il déclare à la société côtoie l'attendu, le conflit qu'il décrit est tout sauf extraordinaire (même si elle est toujours tragique). Il réussit à épingler le discours qui peut être recevable (Derrida 2018 : 21-22), il a le flair pour ce qui, bien qu' « alternatif », est déjà branché sur une réception possible. Intempestif, il l'est, mais cette allure intempestive est calculée dès l'abord.

Dans En finir avec Eddy Bellegueule, l'auteur insiste sur le motif de la fuite car au moment des faits, il s'agissait non pas d'une description, mais d'un engagement, d'un performatif que le livre est censé raconter, expliquer, peut-être aussi justifier dans le sens de donner un sens après-coup. Il insiste d'ailleurs que la fuite, symbole de la naïveté, est venue avant son salut sous forme d'études, d'éducation.

Que dit ce performatif?

Ce que admettent Louis et Eribon, que même si l'individu affirme ne pas appartenir, il ne peut pas effacer toutes les traces, d'où la honte : celle d'être quand même et à la fois quelque chose que l'on ne veut pas, et celle de se sentir coupable de ressentir cette première honte d'appartenir quand même.

Le désir de ne pas appartenir à une communauté suppose qu'on y appartient quand même (et l'inverse). Sinon, ce ne sera pas un désir.

Pourquoi ce désir fait-il des ravages ? Tout d'abord parce qu'il sous-entend évidemment la concaténation interne d'un système, pas-à-pas, et de façon aussi minutieuse que possible, de notions telles que la famille et l'identité.

Car Louis réifie ces concepts, comme une bonne partie des écrivains contemporains de la littérature «identitaire »; il les prend au pied de la lettre comme des « choses » toutes faites, « réelles », qui rappellent les monades de Leibnitz. Ce qui constitue le paradoxe même de cette illusion ancienne de « tout dire », c'est que l'impératif même, celui qui devrait mener à l'aveu public de tout un chacun, établirait un espace public politique totalitaire (Derrida, 2018 : 72). En plus, son projet est tout gidien : il ne détruit pas la famille, il ne veut que fuir la sienne pour pouvoir s'inventer. L'appel foucaldien, qui ne s'épuise pas dans le pathos, illustre le besoin d'un cadre ouvert qui pourrait comprendre ces nouveaux possibles. Et cependant, si, selon Derrida ${ }^{3}$, il n'y a pas d'identité, il n'y

3 Même si les deux, Derrida et Louis, si éloignés soient-ils, ressentent la même rupture violente, l'exclusion de l'éducation et du domaine de la langue française. Derrida 
a que de l'identification, la famille non plus n'a pas d'identité ni d'état (2018 : 35-36), et tout l'édifice identitaire s'écroule.

\section{« La vie n'est pas une biographie ${ }^{4}$ »}

Louis entre en littérature en thématisant la « signature », en changeant son nom impossible, «Eddy Bellegueule » en «Édouard Louis », pour y introduire ses thèmes obsessionnels : la violence, l'injure, la honte et la fuite, déployée dans une folie de la parole confessionnelle.

Ainsi dès l'abord - et la sauce prend pour tous les trois romans -, le roman côtoie-t-il l'autobiographie. Tous les trois romans sont une sorte de récit autotéléologique bien réfléchi avec une note autofictionnalisante produisant un texte réussi, peut-être même trop bien ciblé : tout est à sa place, actuel, au flair de la salvation. Ici il n'est pas question des polémiques en France qui ont condamné le portrait de sa famille et de son milieu d'une perspective « morale $^{5}$ ».

Ce qui est douteux, c'est que la fuite inaugure l'éducation comme la solution finale d'une nouvelle vie, perpétuant un vieux mythe républicain en France de Balzac, qui a donné un rôle impossible de salut à l'éducation d'un Rastignac, qui a quand même eu Vautrin, d'un Julien Sorel, d'un Ivica Kičmanović en Croatie, qui devaient montrer que les jeux ne sont pas faits d'avance. Dans Clartés de tout, de Lacan à Marx, d'Aristote à Mao, Jean-Claude Milner montre qu'aujourd'hui, une telle tentative est vaine, une sorte de passéisme. Louis affirme lui-même qu'il faut de la naïveté pour fuir (2019 : 49). Or, il s'agit aussi d'une tentative de faire le monde plus " proustien », même si il ne l'est pas dans le sens d'un Künstlerroman, un roman de « devenir-écrivain ». Le roman s'achève avec la veste Airness, ridicule, mise à la poubelle, son cadeau de lycée, son désir de porter les « manteaux de monsieur » ou des vestes de laine (2014 : 203) et son rire victorieux à la question «Alors Eddy, toujours aussi pédé ? », enfin riant avec les autres.

L'homosexualité, le contenu scandaleux à l'époque de Sodome et Gomorrhe, ici identitaire, est la raison pour laquelle il fuit, vient à Paris et s'adonne aux études et à l'écriture. Ainsi l'homosexualité devient-elle une existence spécifique, qui prédestine au destin d'un marquis de Custine. Ce qui est pire, c'est que cette homosexualité est toute claire, toute lisse, parfaitement incorporée et incarnée, identifiant son modèle, le Retour à Reims d'Eribon.

Or, l'engagement et la question d'identité personnelle ne sont pas problématiques; le problème est qu'une ligne très fine distingue l'activisme et la science, et l'activisme et la littérature. Si les deux écrivent une socio-analyse

thématise comme Louis la « rupture violente » $(2018: 48)$ ressenti à travers la langue française, et son réinscription dans une école juive d'Alger.

4 Annoncée dans L'enfant d'Ingolstadt, Dernier royaume X (Paris, Bernard Grasset, 2018 : 166), cette phrase va devenir le titre de son livre éponyme de 2019.

5 La revue Marianne et la polémique déplacée de la bienveillance supposée de l'auteur envers la violence, car Reza le criminel est un homme un peu comique et surpris, qui devient violeur comme malgré soi ; tout y ressemble à une farce (Ibid. : 72, 73). 
de l'identité (de l'homosexuel comme minoritaire) et de la classe (ouvrière) du point de vue des écrivains engagés, l'un avec les prétentions scientifiques, l'autre avec les prétentions littéraires, c'est comme si les deux jouent sur le fait de rendre flou cet même espace sur lequel ils écrivent $t^{6}$.

Qu'est-ce qui est problématique ici ? Que la violence doit légitimer la véracité du scientifique et de l'esthétique. Pourquoi la pauvreté garantirait-elle la vérité ? Ce n'est pas pour inaugurer « l'embellissement » de la misère ou l'effacement des exclus, mais le naturalisme est loin d'être une nouveauté. Pourquoi les milieux intellectuels parisiens seraient-ils l'épiphanie de la culture ou l'idéal qu'il faut atteindre ? Pourquoi seraient-ils le « degré zéro » de la référence?

Ils peuvent l'être chez Proust, car il élève un univers très restreint au niveau de l'expérience universelle, d'où son génie. Chez Louis, une telle argumentation ne peut être plausible qu'à ce même public parisien intellectuel qui, comme tout microcosme, juge complaisamment d'après soi et qu'Eribon, par ailleurs, critique. Pourquoi une chose quelconque serait-elle par définition une mesure de vérité vraisemblable? Pourquoi parlons-nous de vérité s'il est question de littérature, qui est en même temps une socio-analyse? La littérature ne sous-entend pas une liste des thèmes obligatoires qui devraient invoquer l'approbation pathétique de tous en les réduisant au silence par les «faits », ici la pauvreté. Et par rapport à quoi ? Ce n'est qu'un trou stérile de l'élitisme et du snobisme bien connu, qui reste un choix légitime de l'individu, mais qui ne parvient pas à se transposer en une expérience possible pour chacun d'entre nous.

Qu'est-ce qui est problématique dans un roman hybride qui combine la socioanalyse et l'autobiographie?

Le fait qu'un tel choix sous-entend la thèse que la vie serait une biographie ${ }^{7}$. Déjà Sartre, dans L'Idiot de la famille (1970, I : 11), son "roman vrai », spécifie que les techniques et les rôles attendent le jeune Gustave Flaubert ${ }^{8}$. Ces derniers, inscrits dans et par le langage, ne sont pas ce qu'est la vie.

Et les romans de Louis, tout en transformant la vie en une narration, en lui inculquant une direction, une causalité, bref, un sens, affichent toutes ses interventions.

La biographie et l'autobiographie sous-entendent rapporter le réel en effaçant non seulement le temps, la diachronie, leur nature langagière, c'est-à-dire

6 Ces textes sont nécessaires, culturels et politiques, car ils montrent le conditionnement mutuel de la subjectivité et de l'espace et de la violence qui en découle, mais ils ne peuvent plus revendiquer pour leur propre compte la classe ouvrière comme leur espace. Les deux, nés pauvres, réussissent (et tant mieux pour eux), s'en détachent via l'écriture et le témoignage sur leur homosexualité et leur appartenance à la classe ouvrière, à laquelle ils n'appartiennent plus. Cela n'amenuise en rien la cruauté et la violence de ce milieu ténébreux, mais amène les auteurs au piège de l'exemplification de la dite vérité - de la pauvreté à l'alcoolisme, du kitsch à la bêtise, du refus, de l'impossibilité et du non-sens de l'éducation à l'incompréhension et le désir de s'évader.

7 Voir Quignard 2019 : 105, 114, 144.

8 Ibid. : 120-122. 
« l'impossible conversion du sens (sensible) au sens (sémantique) » (Quignard 2019 : 118) et littéraire, «A raid on the inarticulate » d'Eliot, (Ibid. : 128) ${ }^{9}$, mais surtout la violence symbolique de ces stratégies : la violence de toute biographie et sa férocité ("maléficiante » Ibid. : 137). Et même si toute lecture est violence (Quignard 2014 : 145), celle de la biographie s'avère être sournoise.

En dénonçant la société et la violence, ce qui est toujours avantageux et nécessaire, Louis la fait sienne. Par ironie, lui aussi promeut le même paradigme en oubliant la leçon foucaldienne sur les pièges de l'impératif assourdissant du discours sur l'identité-sexualité et l'évidence de la question contradictoire, paradoxale que pose l'homosexualité après le féminisme : comment se constituer dans la différence pour demander l'égalité ?

\section{En guise de conclusion : Comment réussir son échec?}

Louis et Eribon semblent contredire la thèse de Quignard, que celui qui écrit, qui pense devrait perdre sa place (2014 : 205). Ils semblent s'être trouvé justement par l'écriture, par la culture. Comme si leur fuite montrait qu'une identité sexuelle mène à une identité culturelle. Elle le peut ou pas, comme toute identité, cela dépend du fait ce que nous sous-entendons par le mot « culture ». Ainsi « écrire dans la langue de l'ennemi », comme disait Genet, de cette "écriture comme un couteau » (Ernaux), devient-il une belle recette.

La violence et la douleur chez Eribon et Louis semblent toujours associés à une tentative sociologique et activiste de témoigner, d'exemplifier en faisant voir les points aveugles de la sociologie bourdieusienne.

Aussi ressentent-ils toujours l'influence néfaste du concept de la sincérité sur la littérature. Encore Gide disait : « il n'y a rien de plus prémédité que la sincérité » ou « on ne peut à la fois être sincère et le paraître ».

La « clarté de tout » n'est pas ici une plateforme pour entrer dans les choix personnels. Elle commence par l'exclusion tout en invoquant l'inclusion, une violence qui ne discrimine pas.

Malheureusement, le jeune et talentueux Louis s'est contenté d'un choix facile au sujet de la façon de traiter le sujet, il a cédé à la tentation de la réalisation rapide d'un positionnement à travers le témoignage qui garantirait la véracité. La bobine est là, le retour à la question de la mimesis.

Tout compte fait, tel Rastignac, Louis a jeté le gant à Paris, et il a réussi en rappelant le symbole de l'impératif balzacien de la réussite, Vautrin.

Naïveté qui « consiste à croire qu'on peut être naïf » (Derrida 2018 : 83), ou pas, nous espérons que Louis va commencer son quatrième roman sur la trace, non pas de "l'idée de l'odeur de pêche » d'Eddy, mais de l'odeur de la pêche, la littérature.

«Malheur à celui qui n'a qu'une histoire à se raconter à lui-même parce qu'elle lui a suffi !» (Quignard 2019 : 157)

9 Ibid. : 86, 128 et 152. 


\section{Bibliographie choisie :}

Derrida, Jacques, Ferraris, Maurizio, Le goût du secret, Entretiens 1993-1995, Paris, Herman Éditeurs, 2018.

Eribon, Didier, Retour à Reims, Flammarion, « champs essais », 2018.

Ernaux, Annie, L'Écriture comme un couteau. Entretiens avec Frédéric-Yves Jeannet, Paris, Stock, 2003.

Louis, Édouard, En finir avec Eddy Bellegueule, Paris, Points, 2014.

Louis, Édouard, Histoire de la violence, Paris, Éditions du Seuil, 2016.

Louis, Édouard, Qui a tué mon père, Paris, Points, 2019.

Milner, Jean-Claude, Clartés de tout, de Lacan à Marx, d'Aristote à Mao (Paris, Lagrasse : Verdier, 2011.

Quignard, Pascal, L'enfant d'Ingolstadt, Dernier royaume X, Paris, Bernard Grasset, 2018.

Quignard, Pascal, Mourir de penser, Dernier royaume IX, Paris, Bernard Grasset, 2014 : 145)

Quignard, Pascal, La vie n'est pas une biographie, Paris, Galilée, 2019.

\section{Nasilje modela : Édouard Louis}

Problematizacija nasilja, sveprisutna u romanima Édouarda Louisa (Raskrstimo s Eddyjem, Povijest nasilja i Tko mi je ubio oca), ovdje će postati platforma za preispitivanje prešućene teze : život nije biografija (Quignard) i hibridizacija žanra koja spaja fikcionalizaciju biografije i socioanalize to ne može promijeniti, usprkos identificiranim modelima : Povratak u Reims Didiera Eribona i Paskalovske meditacije Pierrea Bourdieua. Postoji nasilje koje Louis prokazuje, nasilje društva («geografska fatalnost ») prema « marginalcima », i Foucaultovim « anormalnima », među kojima su i homoseksualci, i postoji nasilje koje on nastavlja - nasilje svake biografije, nasilje riječi i govora i nasilje identiteta. Javlja se pitanje granica angažmana, aktivizma, svjedočenja, neupitne aktualnosti i literature. Bijeg zacijelo nije jedini Louisov projekt.

Ključne riječi: Édouard Louis, Didier Eribon, Pierre Bourdieu, nasilje, sram, hibridizacija 
